\title{
Intervention interdisciplinaire arts/langue : le développement de la culture francophone et d'un rapport positif à la langue
}

\author{
Anne S. Lowe et Monique Richard \\ Université de Moncton
}

\begin{abstract}
Résumé
Cette recherche vise à comprendre quels éléments d'une intervention interdisciplinaire arts/langue ont contribué au développement d'un rapport positif à la langue et à l'enrichissement de l'expérience culturelle des élèves de 6 classes de la 8 e année d'une école francophone du Nouveau-Brunswick. L'étude se penche sur les effets des interventions pédagogiques d'enseignantes et d'enseignants de français, de musique et d'arts visuels. Ces derniers ont conçu un projet d'intervention interdisciplinaire arts/langue où les élèves, selon qu'ils étaient inscrits dans le cours de musique ou dans celui d'arts visuels, ont eu l'occasion de poursuivre les objectifs et d'atteindre les résultats d'apprentissage des programmes d'études de français et de musique, ou de français et d'arts visuels, simultanément, tout en centrant leur attention sur des musiciens et artistes de leur communauté. L'analyse qualitative des données recueillies par entrevues, questionnaire et observations démontre que les interventions arts/langue ont contribué au développement linguistique et culturel des élèves, et que, par contre, certaines conditions sont nécessaires pour assurer le succès de l'expérience interdisciplinaire.
\end{abstract}

\section{Abstract}

The purpose of this study is to understand how an interdisciplinary arts/language project contributed to the linguistic and cultural development of grade 8 students from 6 different classes, in a New Brunswick francophone school. More specifically, the study concentrates on the effects of pedagogical interventions used by teachers of French, Music and Visual Arts. An arts/language project was developed in which students, depending on their choice of Music or Visual Arts classes, were given the opportunity to attain the objectives and reach the learning outcomes of their French and Music, or French and Visual Arts courses, simultaneously, while concentrating on musicians and artists from their own community. The qualitative analysis of data resulting from interviews, questionnaire and observations indicates that the arts/language project contributed to the linguistic and cultural development of the students, and that certain conditions need to be addressed in order to assure a successful interdisciplinary experience. 


\section{Introduction}

La spécificité de l'école de langue française en milieu francophone minoritaire réside dans sa mission qui est de voir à la réussite scolaire des élèves et de contribuer à la sauvegarde de la langue et de la culture francophones (Gérin-Lajoie, 2002). Cette école joue un rôle particulier puisqu'elle doit contribuer à l'épanouissement du potentiel humain des membres de la communauté en offrant aux élèves un lieu de socialisation langagière et culturelle, de construction identitaire, de conscientisation et d'appui à la vie communautaire (Landry et Rousselle, 2003).

Compte tenu de la mission de l'école de langue française en milieu minoritaire, les enseignantes et les enseignants sont appelés à jouer un rôle « d'agent de reproduction linguistique et culturel » (Gérin-Lajoie, 2002, p. 125) ou de «passeur culturel » (Zakhartchouk, 1999, p. 20) afin d'accompagner l'élève dans son cheminement scolaire et identitaire. Ce rôle demande aux enseignantes et aux enseignants de préciser le sens qu'ils accordent aux notions de culture et de reproduction culturelle alors qu'elles ne sont pas clairement définies dans les documents ministériels (Arcand, 2000; Gérin-Lajoie, 2002). En effet, si l'on regarde plus spécifiquement les documents du Nouveau-Brunswick (NB), lieu où s'effectue la présente recherche, les programmes d'études du Ministère de l'éducation contiennent tous un résultat d'apprentissage transdisciplinaire qui touche la culture et le patrimoine: «L'élève devra savoir apprécier la richesse de son patrimoine culturel et affirmer avec fierté son appartenance à la communauté francophone et contribuer à son essor » (Ministère de l'éducation du Nouveau-Brunswick [MÉNB], 2007, p. 2). De plus, les districts scolaires francophones ont adopté des politiques culturelles, communautaires et identitaires qui contiennent des objectifs prescrits parmi lesquels on retrouve, par exemple : « Valoriser auprès des élèves la langue et la culture acadienne et francophone; contribuer à la construction identitaire des élèves; valoriser une pédagogie propre au milieu minoritaire; intégrer les activités artistiques, l'éducation artistique ainsi que la culture acadienne et francophone au curriculum » (District scolaire 01, 2006, p. 2). Pour ce faire, les enseignantes et les enseignants doivent chercher à intégrer une dimension culturelle à leur enseignement en développant des scénarios pédagogiques qui répondent aux éléments essentiels de cette politique. Afin que les élèves s'approprient les éléments de leur langue et de leur culture francophones, l'école doit devenir un espace qui leur offre des vécus socialisants (Landry, Deveau et Allard, 2006) dans leur langue et leur culture. Ces vécus se concrétisent, entre autres, par des 
discussions et des échanges par rapport à l'histoire et à l'actualité de la communauté francophone, par la découverte de produits culturels francophones que sont la musique, le théâtre, la littérature et les divers médias, ainsi que par des rencontres avec des artistes et des gens de la communauté pouvant animer des activités culturelles (Cormier, 2005; Landry, 2003). La question qui se pose alors est de savoir si les efforts déployés par les enseignantes et les enseignants pour intégrer la langue et la culture francophones au curriculum produisent les effets escomptés sur le rapport des élèves à cette langue et à cette culture.

\section{Recension des écrits}

Afin de contribuer à la construction d'une identité francophone forte et engagée ainsi qu'à une motivation langagière autodéterminée pour apprendre le français et en faire usage, la recherche de Deveau, Landry et Allard (2007) montre l'importance d'offrir aux élèves trois types de vécus ethnolangagiers socialisants. Le premier, le vécu enculturant, constitue la base de la socialisation langagière et culturelle (Landry, Deveau et Allard, 2006). II permet de transmettre la langue, les normes, les valeurs et les rôles de chacun dans la société. Un deuxième type de vécu, le vécu autonomisant, est relié à la qualité des contacts interpersonnels et s'inspire de la théorie de l'autodétermination du comportement de Deci et Ryan (2000). C'est ainsi que plus les contacts avec des francophones et la langue française satisfont les besoins d'autonomie, de compétence et d'appartenance de l'individu, plus l'identité francophone sera forte (Deveau, Landry et Allard, 2005). Enfin, le troisième type de vécu, le vécu conscientisant, cherche à développer la conscience critique et le comportement engagé de la personne envers son développement psycholangagier et envers l'épanouissement de sa communauté (Allard, Landry et Deveau, sous presse).

Pour sa part, Cormier (2005) souligne l'importance du développement d'un rapport positif à la langue en créant des occasions signifiantes de production et de réception langagière en français. Selon cette auteure, l'individu qui aime sa langue et qui est fier de son identité résistera plus facilement à l'assimilation et contribuera au rayonnement de sa culture. II est donc important de permettre aux élèves de vivre des activités de socialisation langagière plus informelles au départ qui donnent une image positive à leur langue et à leur culture, pour ensuite passer à des interventions plus formelles en classe.

Puisque la présente recherche s'intéresse de façon particulière aux interventions pédagogiques d'enseignantes et d'enseignants pouvant contribuer au développement culturel et au rapport positif à la langue française, il convient de se pencher davantage sur un des vécus socialisants, soit le vécu enculturant. D'ailleurs, c'est aux enseignantes et enseignants qu'incombe la responsabilité de créer les conditions favorables au vécu enculturant en salle de classe (Gilbert, LeTouzé, Thériault et Landry, 2004) en choisissant des stratégies pédagogiques qui sont les mieux adaptées, non seulement à l'apprentissage du contenu des matières scolaires, mais aussi au développement linguistique et culturel des élèves. Ainsi, afin d'enrichir le vécu enculturant, Landry (2003) propose d'intégrer des activités culturelles dans le curriculum et de favoriser la participation active des élèves afin qu'ils s'approprient les éléments culturels de la francophonie, tout en étant conscientisés aux enjeux de la communauté francophone minoritaire. Selon cet auteur, il faut permettre aux élèves de découvrir des référents culturels francophones en favorisant leur participation aux activités culturelles tout autant qu'à l'organisation et à la préparation de celles-ci. Les élèves doivent donc être actifs, dans un sens participatif, mais aussi, être capables de faire une analyse réflexive tout au long de ces expériences afin de prendre conscience des aspects concrets de ces activités qui 
entretiennent des rapports étroits avec leur histoire et leur réalité d'aujourd'hui (Landry, Allard et Deveau, 2007). Landry (2003) propose en plus que ces activités culturelles intégrées au curriculum se fassent de la communauté vers l'école et vice versa, pour permettre aux élèves de créer des liens affectifs avec leur communauté. En effet, selon l'étude d'Allard, Landry et Deveau (2005), l'élève conscientisé et engagé tend davantage à valoriser la langue et la culture de son groupe, à s'affirmer sur le plan ethnolangagier et à revendiquer ses droits linguistiques. Ainsi, l'intégration d'une dimension culturelle francophone aux apprentissages par une démarche réflexive qui situe les élèves dans la dynamique de la francophonie (Gohier, 2002) contribue à un vécu enculturant qui, en retour, agit sur les compétences langagières et sur le désir de s'intégrer à la communauté francophone (Landry, Allard et Deveau, 2007).

Contribuer au vécu enculturant des élèves implique l'adoption d'une définition de la culture qui tient compte des particularités du contexte dans lequel ils évoluent. Ainsi, puisqu'en milieu scolaire francophone minoritaire il faut contribuer au maintien et à la reproduction, tout autant qu'à l'essor linguistique et culturel francophones, la culture doit refléter la manière d'être de cette communauté qui se donne une vision du futur et un projet d'avenir (Ministère de l'Éducation de l'Ontario [MEO], 1994). Pour renforcer le caractère dynamique et évolutif de la culture en milieu francophone minoritaire, nous nous appuyons sur la définition de Cazabon (2007) qui affirme que la culture constitue le lien entre « la transmission (héritage, tradition, mœurs d'une société) et la construction (participation, engagement, transformation des individus) qui favorise une coproduction entre le récit (la mémoire) et l'agir (la volonté) » (p. 39). La culture, qui englobe toutes les facettes de la personne (Legendre, 2005), véhicule le rapport à soi, aux autres et au monde (Simard, 2002). Elle est le matériau qui lie la personne à son groupe d'appartenance et qui donne un sens à son identité (Gohier, 2002).

Simard (2002) montre que l'intégration d'une dimension culturelle au curriculum se fait par la mise en place de « conditions qui permettent aux élèves de s'approprier, d'intégrer et d'organiser les connaissances culturelles en un tout cohérent, original et personnel, de se situer au sein des problèmes et des réalités complexes de son temps, dans son identité humaine et dans l'histoire » (p. 77). La culture est alors un objet d'apprentissage et un rapport à être analysé (Falardeau et Simard, 2007; Simard, 2002). Donc, pour favoriser la mise en place de conditions qui contribuent au vécu enculturant, l'enseignante ou l'enseignant doit interpréter son rôle de passeur culturel comme l'entend Zakhartchouk (1999), c'est-à-dire comme celui d'un éveilleur qui cherche à faire découvrir les œuvres culturelles, tout en transmettant un regard réflexif sur celles-ci plutôt que de se limiter à un « enseignement transmissif ordinaire » (p. 68). Gohier (2006) affirme que la culture ne peut se transmettre sur un mode d'apprentissage traditionnel qui se limite trop au langage de la rationalité. Gohier (2006), ainsi que Zakhartchouck (1999), indiquent que la personne doit se sentir interpellée pour que le rapport à la culture puisse s'établir. Ces chercheurs concluent que c'est par le langage symbolique des arts que l'individu peut avoir un rapport à la culture qui le lie aux membres de son groupe culturel. Selon Zakhartchouck (1999) ce langage symbolique passe par les productions, les créations et les œuvres de l'humanité, par exemple, la littérature, la peinture, la sculpture, le cinéma et la musique. C'est ainsi, selon Gohier (2006) et Landry (2003), que la connaissance des œuvres d'art, la rencontre avec les artistes ou la rencontre avec des personnes ayant contribué politiquement, spirituellement ou culturellement au développement culturel de sa communauté sont des moyens pouvant favoriser un rapport à la culture qui ne soit pas que cérébral, mais qui contribue à créer le lien d'appartenance affectif avec les membres de sa communauté culturelle. 
De fait, les travaux de Théberge (2006), de Lowe $(2002,2004,2006)$, de Cormier (soumis) et de Simard (2002) indiquent que la participation aux activités artistiques offre aux élèves des occasions de s'approprier la culture francophone et les appuie dans leur cheminement identitaire. En particulier, les recherches de Théberge (2006) en milieu francophone minoritaire révèlent que les activités artistiques offrent des occasions aux élèves de s'approprier la culture francophone, de discuter et de participer à l'élaboration d'activités culturelles qui constituent des lieux d'expression artistique et de rencontre avec la communauté. Ces expériences marquantes appuient les adolescents et les adolescentes dans leur cheminement identitaire en les aidant à mieux comprendre le point de vue de l'autre et à prendre conscience de l'importance de faire des choix en relation avec soi-même. De plus, l'éducation artistique répond aux besoins d'autonomie, de compétence et d'appartenance de l'élève en permettant l'affirmation de soi, la confrontation avec des idées nouvelles et le développement de l'autonomie, de la tolérance et du sentiment d'appartenance à une culture. Ceci contribue non seulement au vécu enculturant, mais aussi au vécu autonomisant alors que les expériences artistiques vécues permettent de satisfaire les besoins d'autonomie, de compétence et d'appartenance de l'individu et renforcent son identité francophone (Deveau, Landry et Allard, 2005).

De son côté, Lowe $(2002,2004,2006)$ soulève que les stratégies pédagogiques interdisciplinaires arts/langue utilisées dans ses recherches permettent non seulement aux élèves d'approfondir leurs apprentissages en français et en arts, mais aussi d'améliorer leurs habiletés créatives et d'augmenter leur motivation envers la langue française et leur fierté dans leurs réussites, tout en développant un rapport positif à leur langue et à leur culture francophones. De plus, l'intervention interdisciplinaire arts/langue a un effet sur l'estime de soi des élèves, particulièrement chez ceux en difficulté d'apprentissage qui se sentent souvent dépourvus devant les exigences scolaires et sociales. Ces résultats sont en accord avec les propos de Landry et Allard (1987) quant à l'importance d'une estime de soi positive comme préalable à l'acquisition d'une identité et d'un sentiment d'appartenance culturelle. À la suite de ses recherches collaboratives auprès des élèves et des enseignantes et enseignants en milieu minoritaire, Lowe $(2002,2004,2006)$ a développé un modèle qui résume les quatre éléments essentiels à la réussite d'expériences d'apprentissage interdisciplinaires arts/langue. L'enseignement, l'apprentissage, le développement professionnel et les conditions environnementales sont des éléments qui interagissent afin de créer un effet spiral (voir annexe I). L'interaction entre ces quatre éléments étant constante, toute intervention sur l'un ou l'autre d'entre eux a un impact sur tous les autres et résulte dans un réaménagement des pratiques pédagogiques et des apprentissages des élèves à un niveau supérieur.

Dans le même sens, Cormier (soumis) démontre l'effet bénéfique d'une intervention pédagogique qui exploite des référents culturels francophones, soit la musique de langue française, sur la construction identitaire et le rapport à la langue d'élèves de la 10e année du Nouveau-Brunswick. Cette intervention, par le biais d'une pédagogie propre au milieu minoritaire, a permis à des enseignantes et enseignants d'intégrer la musique francophone dans leurs cours de français, tout en favorisant une approche communicative qui valorise l'oral et une attitude ouverte envers la variation linguistique. Les élèves ont reconnu l'importance du projet pour leur culture et ont semblé manifester une appréciation générale pour la musique francophone. Cormier (soumis) estime que des initiatives de ce genre tout au 
long du parcours scolaire des élèves leur offriraient des occasions de développer un rapport positif à la langue, une enculturation active et une construction identitaire positive.

Enfin, Simard (2002) soutient que l'éducation artistique joue un rôle dans la valorisation de la diversité culturelle, contribue à la formation de la personnalité et agit sur la construction de l'identité culturelle, tout en permettant l'acquisition des compétences transversales dans d'autres domaines d'apprentissage. Selon ce chercheur, les arts offrent une voie unique qui permet de mieux comprendre le monde et de mieux se connaître.

\section{But de la recherche}

II ressort des propos qui précèdent qu'une intervention pédagogique interdisciplinaire arts/ langue est susceptible de favoriser d'une manière significative le développement d'un rapport positif à la langue et à la culture francophones des élèves en milieu minoritaire. Dans ce contexte, cette recherche vise à déterminer quels éléments d'une intervention interdisciplinaire intégrant les arts visuels, la musique et le français touchent le vécu enculturant de l'élève en contribuant au développement d'un rapport positif à la langue et à l'enrichissement de la dimension culturelle en milieu francophone minoritaire.

Plus spécifiquement, cette recherche vise à comprendre comment ce type d'intervention interdisciplinaire :

- $\quad$ permet aux élèves de vivre leurs apprentissages artistiques et linguistiques;

- $\quad$ permet aux élèves d'être actifs et créatifs plutôt que simplement exposés et sensibilisés à des référents culturels francophones;

- $\quad$ s'avère significatif pour les élèves en ce qui concerne leur désir de s'exprimer en français, d'utiliser des référents culturels francophones et de vivre d'autres expériences reliées à la langue et à la culture francophones.

\section{Méthodologie}

\section{Participante et participants}

Cette étude, réalisée dans une école de langue française du Nouveau-Brunswick, s'est échelonnée sur une période de 8 mois. Elle a impliqué la participation des élèves de 6 classes de la $8^{\mathrm{e}}$ année, dont 3 classes à la session d'hiver 2007 et 3 autres à la session d'automne 2007. S'ajoutent à ces élèves un total de 8 de leurs enseignantes et enseignants: les 6 enseignantes et enseignants de chacune des classes de français, l'enseignante de musique et l'enseignante d'arts visuels. Les enseignantes et enseignants de français avaient entre une année et au-delà de 20 années d'expérience en enseignement tandis que les enseignantes de musique et d'arts visuels en avaient près de 15 années chacune. En ce qui a trait à la participation des élèves, tous suivaient les cours obligatoires de français, mais tous ne suivaient pas la même option d'éducation artistique. Deux des classes participantes étaient formées d'élèves inscrits aux cours de musique, 2 autres classes, d'élèves inscrits en arts visuels et les 2 dernières, d'un mélange d'élèves inscrits en musique et en arts visuels. Bien que tous les élèves de ces classes ont bénéficié de l'intervention interdisciplinaire, la collecte des données à la session d'hiver s'est concentrée sur 12 élèves représentatifs des 3 classes de français. Ceux-ci ont été choisis par leurs enseignantes et enseignants en assurant une diversité dans les niveaux de rendement scolaire : 4 élèves forts, 4 élèves moyens et 4 élèves faibles. À la session d'automne, la collecte des données s'est effectuée auprès des 76 élèves inscrits dans toutes les classes de français. 


\section{Intervention interdisciplinaire}

La présente recherche se situe dans le prolongement d'une étude antérieure réalisée à l'automne 2006 (Lowe et Richard, soumis) et intégrant la musique ainsi que les arts visuels à la création d'un conte collectif. Les 6 enseignantes et enseignants de français, l'enseignante de musique et l'enseignante des arts visuels de la 8 e année ont décidé de reproduire l'intervention développée au cours de cette première étude. Ainsi, les élèves en musique ont été invités à choisir un chanteur, une chanteuse ou un groupe musical francophone, alors que ceux en arts visuels devaient choisir un artiste visuel francophone qui les interpellait. Ils devaient ensuite former équipe avec ceux et celles qui avaient fait les mêmes choix. Tous devaient écrire une synthèse de l'information recueillie sur la biographie de leur artiste (activité de français). De plus, ceux en musique devaient analyser une pièce musicale du chanteur ou de la chanteuse, ou du groupe choisi, puis composer et interpréter un nouveau couplet pour la chanson. Tous les élèves devaient fabriquer un kiosque dans le but de faire une présentation orale aux élèves de la 6 e et de la $7 \mathrm{e}$ année pendant la semaine de la fierté française. Un prix a été accordé pour le meilleur kiosque d'information.

Les tâches confiées aux élèves en arts visuels et l'évaluation de leurs travaux lors de chacune des deux sessions du projet étaient différentes. Pendant la première session (hiver 2007), tout en étant sensibilisés aux artistes visuels acadiens et francophones, les élèves devaient, tout comme leurs pairs en musique, fabriquer un kiosque avec l'information recueillie sur leur artiste. Par contre, pendant la deuxième session (automne 2007), les élèves ont pu approfondir davantage leurs connaissances des artistes visuels acadiens et francophones en créant une œuvre dans le style de l'artiste choisi. Par exemple, une élève a créé un tableau dans le style de Néri DeGrâce. De plus, les productions en musique et en arts visuels ont été évaluées par des juges de l'extérieur et cette évaluation a fait partie des évaluations sommatives en français, en musique et en arts visuels.

\section{Collecte et analyse des données}

Des méthodes qualitatives de collecte et d'analyse des données ont été utilisées pour comprendre le phénomène investigué (Clandinin et Connelly, 2000; Eisenhart et Howe, 1992; Goetz et LeCompte, 1984; Robson, 1993; Strauss et Corbin, 1998). Afin d'assurer la triangulation des données (Bresler et Stake, 1992), une variété de sources, pour chacune des interventions arts/langue, ont été utilisées :

1. l'enregistrement audio d'entrevues semi-structurées conduites avec les enseignantes et les enseignants de français, de musique et d'arts visuels, ainsi qu'avec les 12 élèves choisis parmi les participantes et les participants de la session d'hiver 2007;

2. un journal de bord tenu par la chercheure et où ont été transcrits les propos discutés pendant des entrevues informelles avec les enseignantes et les enseignants, les agentes pédagogiques, le directeur de l'école et les élèves;

3. les notes d'observation et les commentaires de l'assistante de recherche;

4. le matériel didactique utilisé pour les interventions interdisciplinaires.

Aucune entrevue formelle n'a été réalisée avec les élèves à la session d'automne 2007. Cependant, à la suite des interventions arts/langue qui ont eu lieu pendant cette deuxième session, tous les élèves ont répondu par écrit à un questionnaire portant sur les mêmes thèmes que ceux discutés pendant les entrevues avec les élèves à la 
première session (hiver 2007). Le Tableau 1 résume les thèmes abordés lors des entrevues avec les enseignantes et les enseignants, et dans le questionnaire s'adressant aux élèves.

Tableau 1 : Thèmes des entrevues et du questionnaire

\begin{tabular}{|l|l|}
\hline Enseignantes et enseignants & Élèves \\
\hline - Vécu pendant la préparation et le déroulement du & - Description du projet \\
projet & - Description du travail accompli \\
- Développement culturel & - Découvertes culturelles \\
- Résultats d'apprentissage visés et atteints & - Vécu culturel à la maison \\
- Satisfaction du travail des élèves & - Aspects positifs et défis rencontrés \\
- Évaluation & - Valeur de l'intégration des arts au programme de \\
- Motivations des élèves et de l'enseignante ou de & - Mrançais \\
l'enseignant & - Motivation \\
- Points positifs et défis & - Autres \\
\hline
\end{tabular}

Les données ont été analysées en utilisant des techniques et procédures communes en recherche qualitative (Strauss et Corbin, 1998; Paillé et Mucchielli, 2003). Les procédures de codage comprennent : I'analyse microscopique des données, l'identification de codes, de thèmes et de catégories, la classification des catégories à l'aide du logiciel Atlas.ti et l'identification des concepts émergents regroupant les différents éléments du phénomène investigué. Tout au long de cette analyse, des techniques de comparaison constante (Glasser et Strauss, 1967; Strauss, 1987) et de triangulation (Bresler et Stake, 1992; Mathison, 1988) ont été appliquées à toutes les sources de données.

\section{Résultats}

Puisque cette étude cherche à comprendre quels éléments d'une intervention interdisciplinaire arts/langue contribuent à un rapport positif à la langue et au développement culturel des élèves en milieu francophone minoritaire, enrichissant ainsi le vécu enculturant, nous discuterons d'abord des données relatives à ce rapport. Dans un deuxième temps, nous aborderons d'autres catégories émergeant de toutes les sources de données et jugées significatives pour cette étude, en particulier en ce qui a trait à l'apprentissage, à l'enseignement et aux conditions environnementales. Une vue d'ensemble des catégories et des sous-catégories retenues dans cette analyse est présentée à l'annexe 1.

\section{Rapport à la langue et à la culture}

Les activités interdisciplinaires arts/langue visaient l'appréciation et l'exploitation de la musique et des œuvres d'artistes visuels acadiens et francophones, tout en rejoignant des résultats d'apprentissage en français et dans les arts. La nature même des activités visait à sensibiliser les élèves aux produits musicaux et artistiques visuels propres à leur culture. Mais la question demeure la suivante : ces interventions interdisciplinaires ont-elles contribué au 
développement chez les élèves d'un rapport positif à leur langue et à leur culture? En guise de réponse, voyons d'abord ce que révèle l'analyse du discours des élèves, puis de celui de leurs enseignantes et enseignants.

\section{Discours des élèves}

Le discours des élèves montre qu'ils ont découvert des musiciens et des musiciennes, et des artistes visuels acadiens et francophones qu'ils connaissaient peu ou ne connaissaient pas du tout. De plus, ils ont écouté et analysé leur musique ou étudié leurs œuvres d'art dans le détail jusqu'au point d'aimer leurs chansons ou leurs tableaux et d'avoir le goût d'en écouter ou d'en voir davantage. Les élèves ont aussi écrit des lettres aux musiciens et aux artistes de la région, les invitant à venir les rencontrer en salle de classe. Quelques-uns ont répondu à l'appel. Enfin, les élèves faisant partie de la classe d'arts visuels ont eu la chance de visiter quelques artistes acadiens dans leur atelier et de discuter avec eux. Voici un commentaire d'un élève :

J'ai trouvé que le projet était intéressant et très instructif au sujet des différents artistes de la région. Cela m'a aidé à ouvrir mes goûts musicaux et artistiques des artistes de la région. J'ai appris beaucoup sur les artistes locaux et j'ai aussi appris que même les acadiens et les québécois pouvaient avoir de la bonne musique pop et rock en français.

Les élèves n'ont pas seulement été sensibilisés aux musiciens et aux artistes qui faisaient l'objet de leur recherche personnelle, mais ont pu, lors des échanges en classe et pendant les présentations finales, connaître d'autres musiciens et d'autres artistes acadiens et francophones. Quelques élèves témoignent ainsi de leur expérience :

Le projet m'a amené à apprendre à propos d'autres artistes francophones... C'est pas mal ça!

Ça va me motiver d'écouter davantage de la musique française et d'essayer d'écouter d'autre musique francophone...

Quelques élèves ont poussé leur réflexion et tenté d'expliquer la raison pour laquelle ils n'étaient pas attirés vers la musique française auparavant :

Je pensais, mais, c'est de la musique française, ça ne va pas être bien. Mais, ils ont un beau site et leur musique est pas pire du tout... C'est comme bien!

J'ai découvert que beaucoup de la musique d'aujourd'hui c'est pas mal tout anglais puis c'est ce que le monde écoute. Ils n'ont pas d'opinion sur la musique française... Maintenant que je connais plus de musique qui vient de ma culture, j'aime beaucoup ça.

Pour d'autres élèves, la réalisation du projet leur a permis de s'identifier comme acadien et francophone. Une élève affirme : « Je me sentais acadienne comme eux ». Certains autres commentaires des élèves laissent aussi sentir que les interventions arts/langue ont eu un effet sur leur appréciation de la langue française :

J'ai pu comprendre les paroles parce qu'ils chantaient dans notre langue...

Le projet m'a beaucoup intéressé à la musique française et je crois qu'il a aussi motivé beaucoup d'élèves à apprendre et à parler en français.

De plus, les activités ont contribué à sensibiliser les élèves à la diversité de la musique francophone à travers le monde : 
J'ai écouté de la musique africaine en français... II y a beaucoup plus de musique française que je pensais.

Un rapport positif à la culture se révèle aussi dans le fait que certains participantes et participants se sont procurés des œuvres musicales d'artistes francophones dans le but de les écouter avec leurs amies et amis et de les partager avec leurs parents.

En somme, les élèves semblent avoir appris à propos de la culture acadienne et francophone à travers l'activité d'intégration de la musique et des arts visuels dans leur cours de français et semblent s'être identifiés davantage comme étant des acadiens et des acadiennes, et des francophones. Les élèves semblent avoir développé une meilleure appréciation de la langue française et de leur culture, cela contribuant à un rapport positif à celles-ci.

\section{Discours des enseignantes et enseignants}

Le discours des enseignantes et des enseignants abonde dans le même sens que celui des élèves. Ceux et celles qui ont participé aux deux sessions du projet affirment que les interventions ont permis aux élèves d'apprécier davantage leur langue et leur culture. De plus, les élèves ont eu la possibilité de connaître les musiciens et les artistes francophones de la région et de la francophonie en général. Voici un témoignage d'une enseignante de français :

On a énormément touché à la culture dans ce projet. On a valorisé la culture francophone, nos artistes acadiens. Lorsque je passais les kiosques, je remarquais que les élèves étaient vraiment intéressés et qu'ils appréciaient énormément leur langue, leur culture. C'était beau de les voir...

Les enseignantes et les enseignants rapportent que les élèves se sont identifiés aux musiciens et aux artistes visuels francophones de la région et d'ailleurs et ont senti qu'à travers les arts, on raconte une histoire qui est aussi la leur. De plus, ils ont vécu des expériences qui ont contribué au développement d'une certaine fierté d'être acadien et francophone. Les extraits suivants du discours d'enseignantes de français et d'arts visuels appuient ces propos:

Plusieurs m'ont dit que la musique française était la musique québécoise. Ils avaient un blocage là... Ils disaient : « On n'aime pas ça parce que c'est québécois, ce n'est pas acadien. » Un élève m'a dit: «Moi, je veux choisir un artiste de ma culture, de ma région (Shippagan). Je trouve cela super parce que, à travers les œuvres, on se rend compte qu'il y a deux histoires. Puis, c'est notre histoire à chacun de nous. » Ça voulait dire que sa fierté, c'était son petit coin. Il était très fier de dire que Néri DeGrâce venait de par chez-lui.

Le discours des enseignantes et enseignants confirme également que les élèves ont pris le goût d'écouter la musique française et ont éprouvé une certaine satisfaction à la promouvoir. De fait, ils ont enregistré plusieurs chansons françaises sur leur I-POD et ont partagé leurs découvertes avec leurs parents et leurs amies et amis. De plus, les élèves ont aimé jouer la musique de leur musicien et, lors des présentations à la fin du projet, ils ont encouragé les élèves de la $6^{e}$ et de la $7 \mathrm{e}$ année à se procurer leurs œuvres. Une enseignante et l'enseignant de français partagent :

Ils me disent : « J'ai jusqu'à 40 chansons françaises sur mon I-POD. Puis mes parents aiment ça... Mon père a commencé à danser sur une chanson. »

J'ai entendu une élève de 6e dire : « C'est bon ce groupe-là. Est-ce que je peux brûler leur CD? » 
Pour résumer, les enseignantes et les enseignants témoignent que les interventions interdisciplinaires arts/langue ont sensibilisé les élèves à l'appréciation de leur langue et de leur culture, contribuant ainsi au développement d'un rapport positif à celles-ci et enrichissant d'autant le vécu enculturant des élèves. La plupart d'entre eux se sont identifiés aux musiciens et aux artistes visuels acadiens et francophones et, par conséquent, ont renforcé leur identité et leur sens d'appartenance culturelle.

\section{Autres catégories}

Trois autres catégories ont été identifiées suite au regroupement de plusieurs thèmes et sous-thèmes ressortis pendant l'analyse des données : l'apprentissage, l'enseignement et les conditions environnementales. Ces catégories sont discutées dans la section qui suit car elles permettent une compréhension plus profonde et complète de l'impact des interventions interdisciplinaires arts/langue. De plus, ces catégories viennent appuyer en partie le modèle de Lowe (2006) qui stipule que l'apprentissage, l'enseignement, les conditions environnementales et le développement professionnel contribuent au succès des expériences interdisciplinaires arts/langue (Annexe II).

\section{Apprentissage}

Même si l'examen de l'apprentissage réalisé par les élèves dans les matières visées (français, musique et arts visuels) n'apparaît pas d'une façon explicite dans les objectifs de cette recherche, cet élément ressort souvent dans les entrevues avec les élèves et dans les réponses au questionnaire écrit. Ceci est dû au fait que les activités arts/langue ont été développées en lien étroit avec les résultats d'apprentissage des programmes d'études. Pendant la conception initiale du projet interdisciplinaire arts/langue, les enseignantes et les enseignants avaient le souci non seulement de couvrir les aspects culturels contribuant au vécu enculturant de l'élève, mais aussi de toucher les concepts propres à leur matière respective afin de ne pas négliger le contenu du programme d'études. Puisque l'apprentissage semble être un facteur clé au succès de l'expérience interdisciplinaire, cette section abordera les apprentissages spécifiques réalisés en français, en musique et en arts visuels.

\section{Apprentissages réalisés en français}

L'analyse des entrevues et des réponses des élèves au questionnaire écrit révèle que ces derniers ont travaillé plusieurs aspects du français écrit et oral correspondant aux résultats attendus dans le programme d'études et ce, à travers les activités suivantes : l'écriture de la biographie du musicien ou de l'artiste visuel qu'ils ont choisi; l'écriture de la lettre d'invitation à venir en salle de classe adressée aux musiciens et aux artistes visuels; l'écriture du compte rendu de l'expérience à la fin du projet; l'analyse des œuvres musicales et artistiques visuelles et la rédaction d'une appréciation écrite de celles-ci, et, pour les élèves en musique, la composition d'un couplet additionnel pour une chanson. Les élèves ont révisé les règles de grammaire et cherché un vocabulaire riche en corrigeant leurs textes écrits, ont fait la synthèse de l'information recueillie sur la biographie de leur musicien ou artiste visuel, ont été engagés dans la compréhension de la lecture des textes écrits, ont découvert les éléments de la poésie à travers les chants et ont été encouragés à utiliser un français de qualité lors des présentations orales soit dans la salle de classe, soit devant le public. 
Les enseignantes et les enseignants confirment que les élèves ont atteint les résultats d'apprentissage attendus en français par le biais des activités d'intégration d'études de chansons et d'œuvres d'arts visuels au cours de français. Voici deux témoignages de l'enseignante d'arts visuels et d'une enseignante de français :

Pendant la présentation, ils essayaient de sortir leur langage classique qu'ils avaient appris. En parlant des contrastes, des couleurs et de la technique, ils ressortaient les bons termes... Ils essayaient vraiment.

Ils ont développé leur vocabulaire, leur grammaire, leurs habilités en lecture, en écriture et en français oral. Aussi, ils ont travaillé les conjugaisons, la ponctuation, la syntaxe et la calligraphie... Donc, on a touché les objectifs de notre programme.

\section{Apprentissages réalisés en musique}

En ce qui concerne les apprentissages réalisés dans le cadre du programme d'études de musique, les interventions arts/langue ont touché les aspects de l'appréciation de la musique acadienne et francophone. Les élèves ont d'abord découvert certains musiciens et musiciennes acadiens et francophones et ont développé le goût d'en connaître d'autres. En écrivant leur biographie, ils ont acquis des connaissances sur la vie personnelle et professionnelle de l'artiste qu'ils avaient eux-mêmes choisi et ont été sensibilisés à ceux étudiés par leurs pairs. De plus, ils ont eu la chance d'écouter et d'analyser plusieurs œuvres musicales et de les promouvoir auprès de la population étudiante de l'école. Les élèves ont aussi approfondi les concepts de la forme, du style, du timbre et de certaines techniques propres à la composition musicale. Le discours de l'enseignante de musique résume bien les apprentissages réalisés dans cette matière :

Ils ont commencé, pendant la journée découverte, à découvrir les artistes en écoutant leur musique pour choisir celle qui les rejoignait le plus. Ensuite, dans la classe de français, ils ont fait une recherche sur la biographie de leur musicien. Dans ma classe, on a écouté beaucoup de musique acadienne et francophone et ils ont appris comment analyser leurs pièces : différents styles, forme de la chanson, les modulations, les ponts, etc. On a parlé des instruments et comment on arrive à un "build-up" dans la chanson. Puis, je leur ai dit : « Ne vous gênez pas. Ça me ferait plaisir de vous passer mes CD personnels... » J'ai certainement piqué leur intérêt... Ils étaient intéressés puis ils ont accroché sur la chanson. Ça va semer des graines... Même Jonathan, je l'ai mis sur les percussions et il jouait avec le groupe...

\section{Apprentissages réalisés en arts visuels}

Il faut se rappeler que les tâches données aux élèves en arts visuels lors de chacune des deux sessions du projet étaient différentes. En conséquence, les apprentissages en relation avec les résultats attendus dans le programme d'études en arts visuels, pour chacune des sessions du projet, n'ont pas atteint le même niveau de profondeur. Pendant l'expérience vécue à l'hiver 2007, les élèves semblent avoir fait beaucoup de collage à trois dimensions et ont travaillé la couleur et la dimension. Les interventions qui ont eu lieu à l'automne 2007 ont accordé plus d'attention aux arts visuels. De fait, elles ont permis aux élèves de connaître davantage les artistes visuels acadiens et même, de s'identifier à eux en travaillant les aspects techniques de leur création artistique et surtout, en produisant des œuvres dans le style de l'artiste qu'ils avaient choisi. Le témoignage de l'enseignante des arts visuels qui a participé aux deux sessions de l'intervention confirme la qualité supérieure des apprentissages réalisés au cours de la session d'automne: 
Cette fois-ci, (automne 2007), je pourrais dire A+++. Ça touchait directement les artistes visuels... BRAVO! On a vraiment atteint nos objectifs cette fois-ci... Le jeune s'est senti valorisé car il présentait un artiste d'ici et on leur donnait la chance de les rencontrer lors d'un vernissage. Ils ont développé leurs démarches artistiques. Puis, les kiosques étaient supers. Ils ont vraiment compris leurs techniques par rapport aux démarches artistiques des artistes choisis. Oui, cela a vraiment rencontré les objectifs du programme.

\section{Liens établis entre les matières étudiées}

Puisque les interventions arts/langue avaient un caractère interdisciplinaire, il a été jugé important, lors de la collecte des données, de questionner les élèves et les enseignantes et enseignants afin de voir s'ils avaient établis des liens entre les apprentissages en français, en musique et dans le domaine des arts visuels.

Les données révèlent que les élèves ont compris la logique d'intégrer la musique et les arts visuels au cours de français. Ils se sont montrés très conscients du fait que les tâches qu'ils devaient réaliser dans les cours de musique et d'arts visuels leur permettaient en même temps de faire des apprentissages reliés à leurs cours de français écrit et oral. De plus, les élèves semblent avoir apprécié le travail interdisciplinaire et en reconnaissent certains bénéfices. Une élève affirme :

Je trouve que le français est plus facile à apprendre à travers les arts car on apprend plus vite une matière dans une autre qu'on aime beaucoup, comme moi et l'art... On est en train de faire un projet de français qui rejoint les intelligences multiples qui incluent l'art et la musique.

Cependant, il convient de signaler que chaque enseignante et enseignant participant au projet a mis en œuvre les interventions interdisciplinaires dans sa propre salle de classe, indépendamment l'un de l'autre. Les élèves n'ont donc pas eu l'occasion d'observer leurs enseignantes et enseignants travailler en collaboration, ni d'établir des liens de haut niveau entre les apprentissages en français, en musique et en arts visuels comme, par exemple, la reconnaissance de stratégies semblables de composition ou de création utilisées en français et dans les domaines des arts. Toutefois, les élèves ont semblé comprendre l'utilité et les bénéfices de l'intégration de ces trois matières.

De leur côté, les enseignantes et les enseignants des trois matières ne voient plus leur enseignement de la même façon. Ils semblent avoir découvert le potentiel de l'interdisciplinarité et disent vouloir continuer à utiliser cette approche dans le futur. Une enseignante de français rapporte :

Si mes élèves composent un poème et veulent mettre de la musique sur les paroles, ils pourront le faire... Je vois même certains qui feraient un décor pour une chanson qu'ils ont composée, ça pourrait être une publicité pour un groupe musical... II n'y a pas de limites... Puisque ça a été un succès, il faudrait que l'on continue dans cette direction-là.

Pour résumer, les élèves ont fait des apprentissages en lien avec les résultats attendus dans les programmes d'études de français, de musique et d'arts visuels tout au long de l'expérience interdisciplinaire et, tout comme leurs enseignantes et enseignants, ont semblé voir la logique et les bénéfices associés à l'intégration des domaines artistiques au cours de français. 


\section{Enseignement}

Lorsque les enseignantes et les enseignants ont été invités à discuter de leur vécu, ils ont principalement partagé leurs réactions face aux stratégies d'enseignement utilisées pour réaliser les activités interdisciplinaires arts/langue dans leurs cours de français, de musique et d'arts visuels.

Les enseignantes et les enseignants de français ont guidé leurs élèves afin qu'ils découvrent l'information pour la rédaction biographique de leur musicien ou musicienne, ou artiste en arts visuels et les ont encouragés à utiliser un vocabulaire riche et un français correct. L'une d'elles a insisté sur l'authenticité de la tâche et le soutien requis; elle a « demandé aux élèves de pratiquer leur présentation en classe comme si le juge et les élèves de l'école étaient présents». Elle a partagé avec eux des «petits outils pour pouvoir réaliser leurs projets». Une autre enseignante aurait préféré réaliser le projet uniquement avec les élèves de sa classe. Cette enseignante encourage l'apprentissage centré sur l'élève : « J'ai toujours encouragé mes élèves à partager leurs idées personnelles... J'aime que les idées viennent d'eux. Je leur propose de mettre des idées au tableau, de faire un remue-méninges... ». Une autre enseignante encore insiste sur son souci de promouvoir l'amour de la langue française et de trouver des moyens de « donner le goût d'écrire et de parler français... » à ses élèves en leur proposant « des projets qui les rejoignent ». Elle affirme que les activités d'écriture de la biographie, de la lettre à l'artiste et du compte rendu à la fin du projet ont permis aux élèves d'ajouter une touche personnelle à leur apprentissage : «Ils ont fait leur propre projet, ils se sont épanouis là-dedans ».

Pour les deux autres matières, l'enseignante des arts visuels s'est perçue comme « un guide » afin, en particulier, « d'aider les élèves au point de vue technique, etc. » L'enseignante de musique s'est « préparée pour faire le projet avec ses groupes » en leur donnant l'occasion de vivre le processus d'appréciation de la musique (l'écoute, la reconnaissance des différents styles, l'analyse d'une chanson : refrain, couplets, tonalités, modulations) dans un autre contexte musical, avant de débuter les activités reliées au projet interdisciplinaire proprement dit. Tout en « permettant une certaine liberté aux élèves », elle voulait s'assurer qu'ils aient le bagage nécessaire pour accomplir avec succès leur travail.

En somme, l'analyse des données concernant les stratégies d'enseignement utilisées par les enseignantes et enseignants indiquent qu'une pédagogie de la découverte et de la création personnelle centrée sur l'élève semble contribuer au succès d'un projet interdisciplinaire arts/langue).

\section{Conditions environnementales}

Les données qui ont été classées dans la catégorie des conditions environnementales se divisent en trois sous-catégories touchant la motivation suscitée chez les élèves et les enseignantes et les enseignants par les activités reliées au projet d'intervention interdisciplinaire, les dimensions de collaboration et de communication entre les élèves et entre les enseignantes et enseignants, de même que les contraintes reliées au temps.

\section{La motivation}

Les résultats révèlent que les facteurs qui contribuent à la motivation des élèves au cours de l'intervention interdisciplinaire diffèrent quelque peu de ceux qui motivent leurs enseignants et enseignantes. 


\section{La motivation des élèves}

La motivation a été reconnue par tous les participantes et participants comme un élément qui a contribué au succès de l'expérience. Les élèves, par ailleurs très motivés en général par les cours de musique et d'arts visuels, ont affirmé que le fusionnement des matières leur a permis d'apprendre «en s'amusant ». De plus, les tâches proposées aux élèves ont semblé les motiver naturellement comme le souligne une enseignante de français : « lls étaient à la tâche... Je n'avais pas besoin de les inciter à travailler. »

Certains élèves ont particulièrement aimé les activités se rattachant à la rencontre des musiciens, des musiciennes et des artistes visuels francophones, au travail d'équipe, à la fabrication des kiosques, à la présentation orale et aux journées découvertes. D'autres se sont sentis frustrés par certaines de ces mêmes activités. Ces frustrations ont été souvent causées par le manque de participation de certains membres de l'équipe ou par le manque d'organisation de la part des enseignantes et des enseignants. Certaines situations problématiques lors des présentations orales, c'est-à-dire le manque de discipline de la part des élèves pendant les présentations au gymnase (hiver 2007) ou la répétition des présentations aux 6 e et 7 années (automne 2007) en ont démotivé certains. Toutefois, la nouveauté du projet a ajouté du piquant à l'expérience d'apprentissage et a produit des résultats intéressants pour plusieurs. Les enseignantes et les enseignants témoignent qu'à la suite des interventions arts/langue, la majorité de leurs élèves ont écouté davantage de musique française et ont pris goût à faire de la lecture en français. De plus, les élèves ont manifesté leur hâte de s'engager dans un autre projet semblable. Les interventions arts/langue semblent avoir aussi rejoint les intérêts et les forces des élèves, y compris ceux et celles en difficulté d'apprentissage. L'enseignante des arts visuels rapporte :

Cela a été les chercher... Je pense en particulier à une élève qui pourrait décrocher. Je peux même en voir plus qu'une... Elle a trouvé une artiste qui lui ressemblait. Elle s'est rendue compte que cette artiste n'avait pas eu une enfance facile et alors, elle devrait la comprendre... Elle a tellement travaillé... C'était beau de la voir. Ça m'a énormément touchée parce que c'en est une qui aurait dit: " Ça ne m'intéresse pas ». Elle est allée rencontrer l'artiste et a réalisé qu'elle pouvait s'exprimer par les arts.

Les élèves ont aussi été motivés par le soutien des médias et des parents. Un journaliste de Radio Canada est venu les interviewer et les parents ont eu la chance de participer aux présentations finales. Ce soutien de la communauté semble avoir contribué à la motivation des élèves et de leurs enseignantes et enseignants envers ce projet. De plus, les activités d'évaluation sommative permettant d'obtenir soit un prix (hiver 2007), soit une note finale attribuée par les membres du jury (automne 2007) semblent avoir incité les élèves à se dépasser et à prendre conscience de l'importance du produit final.

\section{La motivation des enseignantes et des enseignants}

Tout comme les élèves, les enseignantes et les enseignants ont été motivés par le projet. Voici le témoignage d'une enseignante de français :

J'ai adoré cela une fois que je savais ce que je devais faire. C'était très bien... Et intéressant. J'ai beaucoup aimé l'intégration de l'art, de la musique et du français. Tu sais, ils n'aiment pas le français en partant. Alors, je trouve que c'est intéressant et différent... Au lieu de toujours avoir un livre, une lecture puis une compréhension en lecture. En tout cas, dans ma classe, ils écoutent plus de musique française et aiment lire... Je suis contente. 
Ce témoignage révèle que la motivation des enseignantes et des enseignants est reliée, d'une part, à leur sentiment de compétence dans l'utilisation des nouvelles stratégies d'intervention interdisciplinaire et, d'autre part, dans l'intérêt accru qu'ils perçoivent chez leurs élèves envers la matière à apprendre. De plus, ce témoignage indique que le projet interdisciplinaire arts/langue a été vécu comme un processus d'apprentissage pédagogique pour les enseignantes et les enseignants, et donc, a contribué à un perfectionnement professionnel ancré dans leur vécu.

En somme, malgré la reconnaissance de plusieurs éléments qui semblent avoir contribué à la motivation des élèves et des enseignantes et des enseignants, l'analyse des données révèle que le manque d'organisation pendant la planification, le déroulement et la présentation finale du projet de la part des enseignantes et enseignants ont contribué à certaines frustrations. Toutefois, les bénéfices de l'expérience ont été jugés plus importants que les difficultés rencontrées en cours de route. II est donc permis de conclure que la motivation de tous les participantes et participants de l'étude a contribué au succès de l'expérience.

\section{La collaboration et la communication}

Encore une fois, les données relatives aux dimensions de la collaboration et de la communication révèlent des préoccupations différentes selon qu'il s'agit des élèves ou des enseignantes et enseignants.

\section{Collaboration et communication entre élèves}

En ce qui a trait à la collaboration et à la communication, les données indiquent que la majorité des élèves ont aimé travailler en équipe avec leurs pairs de la même classe, de même qu'avec ceux des autres classes de la huitième année : « J'ai aimé travailler avec les personnes des autres classes... Ça va toujours mieux à deux ou plus... On a plus d'idées » affirme un élève.

Toutefois, certains autres élèves ont vécu quelques difficultés en travaillant en équipe. L'un d'entre eux rapporte :

Dans notre équipe, il y avait certaines personnes qui travaillaient beaucoup et d'autres qui ne travaillaient pas du tout. On était trop un grand groupe (6 personnes). Deux ou trois personnes auraient été préférables... Aussi, on avait de la misère à s'accorder...

Ces propos indiquent que les difficultés rencontrées par les élèves semblent être reliées à deux aspects importants à considérer dans la planification du travail en équipe, notamment le nombre d'élèves assignés à chacun des groupes et la prise en compte des rapports affinitaires entre les membres du groupe.

\section{Communication et collaboration entre enseignantes et enseignants}

Les enseignantes et les enseignants, de leur côté, ont placé la collaboration et une bonne communication parmi les éléments primordiaux pour le succès d'une intervention interdisciplinaire tel que celle-ci. En parlant de leurs expériences pendant les deux interventions arts/langue, tous ont indiqué que le manque de communication et de collaboration entre eux a été la source des plus grandes difficultés rencontrées pendant le projet. Le discours d'une enseignante de français appuie cette affirmation :

J'ai trouvé la journée de planification pénible. Ce n'est pas tout le monde qui a la même vision et les mêmes attentes. Ce n'est pas évident de prendre des décisions à cinq... Encore là, il y a eu un 
manque de communication entre les membres de l'équipe. On s'est réuni quelques fois, mais ce n'était pas assez... Chacun de nous travaillait de son côté sans savoir trop où on s'en allait... Par exemple, à une des dernières réunions, certaines enseignantes ont proposé d'abolir les kiosques... J'ai dit : " Quoi? On a déjà dit aux élèves qu'il allait y avoir des kiosques. On ne peut pas leur dire autre chose... » II aurait fallu que l'on communique davantage.

De plus, le manque de leadership pendant l'expérience semble avoir été un facteur central aux problèmes de collaboration et de communication. L'enseignante de musique affirme :

Ça nous aurait pris un leader pour organiser les rencontres. On aurait pu se partager les tâches et s'assurer que le projet se déroulait tel que prévu...

En outre, le manque de communication entre les enseignantes et enseignants à causé des problèmes organisationnels qui ont été ressentis par les élèves, comme nous l'avons noté précédemment. Cependant, malgré les difficultés rencontrées, tous affirment vouloir revivre l'expérience car ils sont convaincus que ces problèmes peuvent se corriger. Une enseignante de français déclare :

C'était nos premières expériences. Maintenant, nous savons ce que nous devons faire pour régler ces problèmes... Je suis donc prête à m'embarquer encore si l'occasion se présente car le projet s'est bien passé malgré tout.

\section{Contraintes de temps}

Toutes les sources de données, y inclus celles reliées au discours des élèves, indiquent que le facteur temps a constitué une contrainte majeure contribuant en partie aux difficultés de collaboration et de communication vécues par tous les participants. Les enseignantes et les enseignants ont déploré le manque de temps pour se rencontrer afin de planifier et de gérer les interventions, tout autant que l'échéancier trop court pour terminer les projets des élèves et pour rencontrer les résultats d'apprentissage attendus dans les programmes d'études pour les trois matières. Ils auraient souhaité avoir plus de temps pour discuter des problèmes rencontrées pendant le processus afin de les solutionner. Ils proposent, dans le cas où des projets interdisciplinaires similaires seraient mis en œuvre à l'avenir, que des rencontres hebdomadaires soient organisées et qu'un échéancier plus réaliste soit déterminé. Voici le témoignage de l'enseignante des arts visuels :

C'était le manque de temps... On a été poussé par le temps... Fallait que tout soit terminé pour la semaine de la fierté française. On aurait dû avoir plus de temps pour se rencontrer, pour discuter... J'ai rencontré plusieurs de mes résultats d'apprentissage, mais j'aurais pu en faire plus avec plus de temps...

En somme, les participantes et participants de l'étude confirment que les conditions qui ont suscité une forte motivation chez les enseignantes et enseignants telles que la qualité et la nouveauté des interventions interdisciplinaires arts/langue et l'intérêt accru des élèves envers le projet, leur ont permis de surmonter les difficultés rencontrées au niveau de la communication et de la collaboration, tout autant que les contraintes du temps.

\section{Résumé et conclusion}

Cette étude cherchait à comprendre quels éléments d'une intervention interdisciplinaire arts/langue ont contribué au développement d'un rapport positif à la langue et à l'enrichissement de l'expérience culturelle des élèves 
de la $8^{e}$ année d'une école francophone en milieu linguistique minoritaire. Elle se penchait de façon particulière sur les interventions pédagogiques qui pourraient contribuer au vécu enculturant des élèves.

Les résultats indiquent que l'intégration des activités d'analyse de chansons et d'œuvres d'arts de musiciens et musiciennes, et d'artistes visuels acadiens et francophones au cours de français a sensibilisé les élèves à des créations musicales et artistiques propres à leur culture et leur ont donné le goût d'en écouter ou d'en voir davantage. De plus, un grand nombre d'élèves se sont identifiés à la culture acadienne et francophone et ont développé une appréciation de la langue française, donc, ont vécu un sentiment de fierté acadienne et francophone. Leurs enseignantes et enseignants témoignent que les activités interdisciplinaires arts/langue ont permis d'aborder les aspects culturels de leur programme d'études, c'est-à-dire, de promouvoir et de vivre les valeurs de la société acadienne et francophone et de sensibiliser les jeunes à l'importance de la langue et de la culture francophones. Les élèves semblent avoir compris que, par le biais des arts, ils peuvent s'exprimer et raconter leur histoire, donc, que les arts sont un moyen privilégié d'expression personnelle et sociale. Les enseignantes et les enseignants affirment alors que les interventions arts/langue ont contribué au développement linguistique et culturel de leurs élèves, favorisant ainsi un rapport positif à leur langue et à leur culture.

Nous constatons aussi que parmi les trois vécus socialisants (Deveau, Landry et Allard, 2007) qui contribuent à la construction d'une identité francophone engagée et à une motivation langagière autodéterminée pour apprendre le français et en faire usage, les interventions interdisciplinaires arts/langue ont non seulement enrichi le vécu enculturant en touchant différents aspects de la langue et de la culture francophones et acadiennes, mais semblent en plus avoir renforcé certains éléments du vécu autonomisant. En effet, le fait de permettre aux élèves de participer activement au processus de créativité, de partager leurs idées, de construire ensemble et de faire des choix selon leurs intérêts et habiletés, a contribué au vécu autonomisant. Toutefois, il serait important d'effectuer une recherche qui exploite ce vécu d'une manière plus explicite avant de conclure que ce genre d'intervention interdisciplinaire enrichit le vécu autonomisant. Quant au vécu conscientisant, il semble avoir été peu touché dans cette intervention. Les discussions et les échanges entourant les différents aspects des projets réalisés par les élèves ne cherchaient pas nécessairement à développer une conscience critique et un comportement engagé envers l'épanouissement de sa communauté francophone (Allard, Landry et Deveau, sous presse). Les activités visaient plutôt une sensibilisation à la langue et à la culture et le développement d'une fierté et d'un rapport positif à la langue, mais elles n'ont pas nécessairement mené les élèves à prendre conscience des enjeux de la communauté francophone minoritaire.

L'analyse des données a également permis d'identifier trois grandes catégories de préoccupations qui ajoutent à la compréhension des représentations que se font les participantes et participants des conditions de succès d'une intervention interdisciplinaire arts/langue : l'apprentissage, l'enseignement et les conditions environnementales. Lors des deux années du projet, les élèves, tout autant que leurs enseignantes et enseignants, reconnaissent que l'intervention leur a permis de développer leurs compétences en français, en musique et en arts visuels. Pour leur part, les enseignantes et les enseignants témoignent que les stratégies d'apprentissage et d'enseignement centrées sur l'élève telles que la pédagogie de la découverte et de la création personnelle, semblent avoir contribué au succès de l'expérience. De plus, trois conditions environnementales qui favorisent un processus de travail sain et un certain 
enthousiasme tout au long de l'intervention interdisciplinaire, ont été identifiées. La première montre, d'une part, que les élèves ont été hautement motivés par la nature des activités arts/langue, par la nouveauté de l'approche interdisciplinaire, par l'évaluation sommative du produit final et par le travail d'équipe. D'autre part, les enseignantes et les enseignants se sont investis tout au long de l'intervention interdisciplinaire car ils se sont sentis de plus en plus compétents au fur et à mesure qu'ils vivaient cette expérience d'enseignement et qu'ils voyaient, chez la plupart de leurs élèves, un engagement et un enthousiasme pour les activités planifiées, et un progrès constant en français, en musique et en arts visuels. La deuxième met l'accent sur l'importance pour les concepteurs d'un tel type d'intervention arts/langue de créer les conditions qui favorisent une collaboration et une communication efficaces entre les élèves et entre les membres du corps enseignant. La troisième réfère au temps nécessaire pour réaliser un tel projet d'intervention. Les enseignantes et les enseignants indiquent que le manque de temps pour se rencontrer et discuter de la planification, de l'organisation et du bon déroulement du projet a été l'un des plus grands défis à relever.

Il est important de noter que les trois grandes catégories identifiées dans cette recherche appuient en partie le modèle de Lowe (2006) qui stipule que l'apprentissage, l'enseignement, les conditions environnementales et le développement professionnel contribuent au succès des expériences interdisciplinaires arts/langue (Voir annexe II). Même si les références au développement professionnel n'ont pas été mentionnées assez souvent dans les sources de données pour justifier la création d'une catégorie d'analyse supplémentaire, il demeure que certains propos des enseignantes et des enseignants indiquent que l'intervention a été très enrichissante pour leur formation personnelle et professionnelle.

En somme, l'analyse des données recueillies au cours de cette intervention interdisciplinaire arts/langue permet de conclure, qu'en plus de contribuer au développement linguistique et culturel des élèves et d'un rapport positif à la langue et à la culture francophones en milieu minoritaire, les expériences vécues à l'hiver 2007 et à l'automne 2007 ont favorisé chez les élèves l'atteinte de certains résultats d'apprentissage en français, en musique et en arts visuels, ainsi qu'un enseignement où la pédagogie de la découverte et de la création personnelle a été valorisé, tout en permettant d'identifier quelques conditions environnementales essentielles au succès d'une telle intervention. Un élève résume bien en quoi son expérience a été bénéfique :

Lorsque je partage mes idées, je parle maintenant en français. En écoutant la musique française dans les cours de musique, de français ou à la maison, j'apprécie ma langue et ma culture. Les éléments de ma langue et de ma culture que je trouve à travers la musique est que le français est une très belle langue artistique et attirante... Bien, je trouve que l'apprentissage du français est définitivement plus intéressant lorsque je le fais à travers les arts... J'ai adoré le projet et j'ai hâte d'en vivre un autre bientôt.

Cependant, malgré le fait que les enseignantes et les enseignants, et les élèves, semblent avoir apprécié cette intervention interdisciplinaire et ont exprimé le désir de revivre ce genre d'expérience, il faudrait voir si à long terme, cette motivation demeure. II conviendrait donc de vérifier dans des recherches ultérieures, si ce type de projet a des retombées à long terme. De plus, même si Deveau, Landry et Allard (2007) insistent sur l'importance de développer trois types de vécus socialisants, cette recherche ne portait que sur un aspect particulier, soit celui du vécu enculturant. Des recherches futures pourraient donc être planifiées pour intégrer aux stratégies d'intervention arts/langue contribuant au vécu enculturant, des stratégies pouvant aussi contribuer aux vécus autonomisant et 
conscientisant des élèves. Toucher ces trois vécus simultanément permettrait à l'élève de développer une conscience critique et un comportement engagé envers son développement psycholangagier et envers l'épanouissement de sa communauté.

\section{Références}

Allard, R., Landry, R. et Deveau, K. (sous presse). Le comportement ethnolangagier engagé en contexte minoritaire. Dans R. Bergeron, L. Lafontaine et G. Plessis-Bélair (dir.), La place des savoirs oraux dans le contexte scolaire d'aujourd'hui. Québec : Presses de l'Université du Québec.

Allard, R., Landry, R. et Deveau, K. (2005). Conscientisation ethnolangagière et comportement engagé en milieu minoritaire. Francophonies d'Amérique, 20, 95-109.

Arcand, D. (2000). Culture, identité et fonction culturelle de l'école francophone : représentations chez les intervenants au conseil scolaire francophone de la Colombie-Britannique. Mémoire de maîtrise inédit, Université Simon Fraser, Colombie-Britannique.

Bresler, L. et Stake, R. (1992). Qualitative research methodology in music education. Dans R. Colwell (dir.), The handbook of research on music teaching and learning (p. 75-90). New York : MacMillan.

Cazabon, B. (2007). Langue et culture. Unité et discordance. Sudbury : Éditions Prise de parole.

Clandinin. F. et Connelly, M. (2000). Narrative inquiry : Experience and story in qualitative research. San Francisco, CA : JosseyBass.

Cormier, M. (soumis). Quand des élèves adolescents de milieux minoritaires ont l'occasion d'écouter la musique francophone. Dans S. Lafrenière (dir.) Collectif. Sudbury : Éditions Prise de parole.

Deci, E. L. et Ryan, R. (2000). Self-determination theory and the facilitation of intrinsic motivation, social development, and wellbeing. American Psychologist, 55 (1), 68-78.

Deveau, K., Landry, R. et Allard, R. (2007). Autodéfinition et engagement identitaire : variables médiatrices d'une motivation langagière autodéterminée. Dans K. Deveau, Identité francophone en milieu minoritaire : Définition et validation d'un modèle empirique de la construction identitaire et de l'autodétermination langagière (p. 112-145). Thèse de doctorat inédite, Université de Moncton.

Deveau, K., Landry, R. et Allard, R. (2005). Au-delà de l'autodéfinition : composantes distinctes de l'identité ethnolinguistique. Francophonies d'Amérique, 20, 79-93.

District scolaire 01 (2006). Politique culturelle, communautaire et identitaire. Dieppe: District scolaire 01.

Eisenhart, M. A. et Howe, K. R. (1992). Validity in educational research. Dans M. D. Lecompte, W. L. Millroy et U. J. Preissie (dir.), The handbook of qualitative research in education (p. 643-680). San Diego, CA : Academic Press.

Falardeau, E. et Simard, D. (2007). Rapport à la culture et approche culturelle de l'enseignement. Revue canadienne de l'éducation, 30(1), 1-24.

Gérin-Lajoie, D. (2002). Le rôle du personnel enseignant dans le processus de reproduction linguistique et culturelle en milieu scolaire francophone en Ontario. Revue des sciences de l'éducation, 28 (1), 125-146.

Gilbert, A., LeTouzé, S., Thériault, J. et Landry, R. (2004). Le personnel enseignant face aux défis de l'enseignement en milieu minoritaire francophone. Ottawa : Fédération canadienne des enseignantes et des enseignants; Centre interdisciplinaire de recherche sur la citoyenneté et les minorités; Moncton : Institut canadien de recherche sur les minorités linguistiques.

Glasser, B. G. et Strauss, A. L. (1967). The discovery of grounded theory. Strategies for qualitative research. New York : Aldine de Bruyter.

Goetz, T. P. et LeCompte, M. D. (1984). Ethnography and qualitative design in educational research. Orlando, FL : Academic Press.

Gohier, C. (2006). Éducation et fragmentation identitaire : à la recherche d'un centre de gravité. Éducation et francophonie, 34 (1), 148-161.

Gohier, C. (2002). La polyphonie des registres culturels, une question de rapports à la culture. L'enseignant comme passeur, médiateur, lieur. Revue des sciences de l'éducation, 28 (1), 215-236. 
Landry, R. (2003). Pour une pédagogie actualisante et communautarisante en milieu minoritaire francophone. Dans R. Allard (dir.), Actes du colloque pancanadien sur la recherche en éducation en milieu francophone minoritaire : Bilan et prospectives, ( $p$. 135-156). Québec, QC : ACELF; Moncton, NB : Centre de recherche et de développement en éducation (CRDE).

Landry, R. et Rousselle, S. (2003). Éducation et droits collectifs : au-delà de l'article 23 de la Charte. Moncton : Éditions de la Francophonie.

Landry, R. et Allard, R. (1987). Étude du développement bilingue chez les Acadiens des provinces maritimes. Dans R. Théberge et J. Lafontant (dir.) Demain, la francophonie en milieu minoritaire (p. 63-111). Saint Boniface, MB: Centre de recherche du Collège de Saint-Boniface.

Landry, R., Deveau, K. et Allard, R. (2006). Langue publique et langue privée en milieu ethnolinguistique minoritaire : les relations avec le développement psycholangagier. Francophonies d'Amérique, 22, 167-184.

Landry, R., Allard, R. et Deveau, K. (2007). Profil sociolangagier des élèves de $11^{e}$ année des écoles de langue française de I'Ontario. Moncton : Institut canadien de recherche sur les minorités linguistiques.

Legendre, R. (2005). Dictionnaire actuel de l'éducation, 3e édition. Paris : Guérin.

Lowe, A. (2006). The Co-Construction of a Music/Arts/Language Learning Model. Journal of Border Educational Research, 3(2), 1931.

Lowe, A. (2004). Intégration des arts au programme de français en milieu francophone minoritaire : bénéfices pour tous les apprenants et apprenantes. Francophonies d'Amérique, 18, 5-19.

Lowe, A. (2002). Recherche collaborative musique/arts/langue en milieu linguistique minoritaire : apprentissage interdisciplinaire et développement de l'identité. Revue canadienne de linguistique appliquée, 5(1-2), 93-116.

Lowe, A. et Richard, M. (soumis). Éducation à la culture par le biais des arts en milieu francophone minoritaire. Revue canadienne de linguistique appliquée.

Mathison, S. (1988). Why triangulate? Educational Researcher, 17(2), 13-17.

Nouveau-Brunswick, Ministère de l'éducation (2007). Programme d'études en musique. Fredericton : Ministère de l'Éducation.

Ontario, Ministère de l'Éducation (1994). Investir dans l'animation culturelle. Toronto : Ministère de l'Éducation.

Paillé, P. et Mucchielli, A. (2003). L'analyse qualitative en sciences humaines et sociales. Paris : Armand Colin.

Robson, C. (1993). Real world research : A resource for social scientists and practitioner researchers. Oxford : Blackwell.

Simard, D. (2002). Contribution de l'herméneutique à la clarification d'une approche culturelle de l'enseignement. Revue des Sciences de l'éducation, 28(1), 63-82.

Strauss, A. L. (1987). Qualitative analysis for social scientists. Cambridge : Cambridge University Press.

Strauss, A. et Corbin, J. (1998). Basics of qualitative research. Thousand Oaks, CA : Sage Publications.

Théberge, M. (2006). Construction identitaire et éducation théâtrale dans un contexte rural franco-ontarien. Éducation et francophonie, 34(1), 133-147.

Zakhartchouk, J.-M. (1999). L'enseignant, un passeur culturel. Paris : ESF. 


\section{Annexe I}

Catégories et sous-catégories

I. CULTURE

a) Rapport positif à la langue

- Expression personnelle

- Engagement

- Sens d'appartenance

- Valorisation

- Fierté de la langue

- Création personnelle

- Impact

II. APPRENTISSAGE

a) Français

- Contenus d'apprentissage

- Rapport positif à la langue

d) Liens entre les apprentissages

III. ENSEIGNEMENT

\section{a) Planification}

d) Intégration des matières

III. CONDITIONS

a) Motivation

- attentes

- appui de l'école et du disctrictl b) Enculturation

- Média

- Sens d'appartenance

- Pouvoir des arts

- Impact b) Arts Visuels

- Contenus d'apprentissage

e) Apprentissage transdisciplinaire

b) Choix

c) Stratégies d'enseignement

c) Musique

- Contenus d'apprentissage

f) Évaluation b) Collaboration/communication

c) Temps

- participation

- leadership

- flexibilité

- horaire

- individualité

- changements 


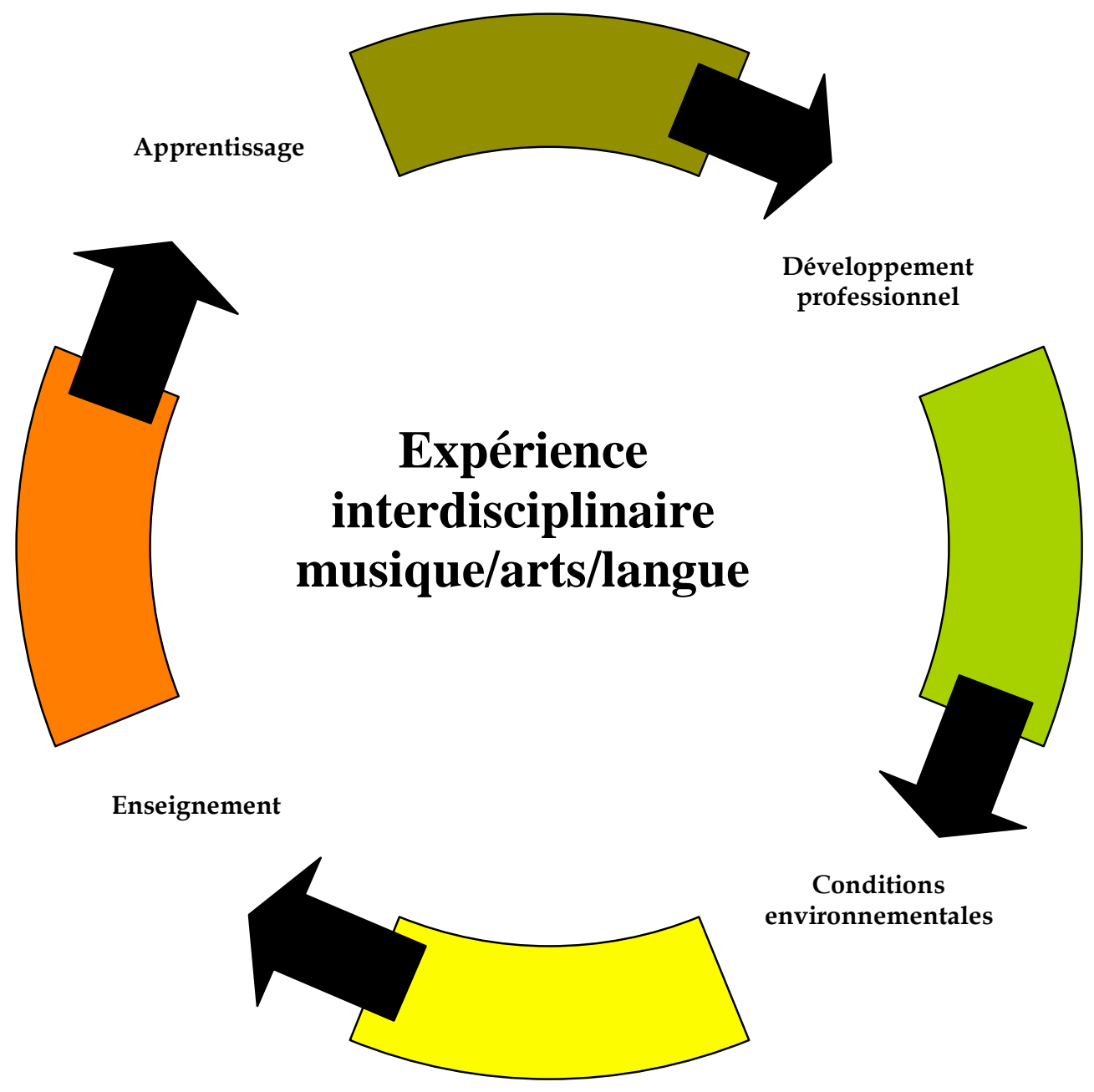

